\title{
Multiple Access and Two-way Channels with Energy Harvesting and Bi-directional Energy Cooperation
}

\author{
Kaya Tutuncuoglu Aylin Yener \\ Wireless Communications and Networking Laboratory, Electrical Engineering Department \\ The Pennsylvania State University, University Park, PA 16802 \\ kaya@psu.edu yener@ee.psu.edu
}

\begin{abstract}
This paper considers the multiple access and twoway channels with energy harvesting transmitters that can cooperate by transferring energy to each other. Specifically, the jointly optimal transmit power allocation and energy transfer policies that achieve the sum-capacity in both models are found. These models were first considered in previous work with unidirectional energy transfer, and optimal policies were found using a two-dimensional water-filling algorithm. In this paper, the bi-directional extension of the energy cooperation model is considered. Using an equivalent energy transfer efficiency representation, it is found that in the optimal policy, a node cannot simultaneously send and receive energy. It is shown that a class of power policies termed procrastinating policies include at least one optimal policy, leading to the insight that the resulting power allocation problem can be solved by a one-dimensional directional water-filling algorithm. It is observed that in the multiple access channel, a node either transfers no energy, or transfers all of its energy to a single user to achieve sum-capacity. For the two-way channel, the optimal policy is found to have a directional water-filling interpretation with two non-mixing fluids whenever optimal energy transfer is non-zero.

Index Terms-Energy harvesting, energy cooperation, energy transfer, optimal power allocation, wireless networks, multiple access channel, two-way channel.
\end{abstract}

\section{INTRODUCTION}

We consider communication systems where transmitters are energy harvesting and can cooperate by transferring energy to each other [1], [2]. In particular, we find the optimal transmit power and bi-directional energy transfer allocations that achieve the sum-capacity of the system in the multiple access channel (MAC) and the two-way channel (TWC).

Energy harvesting networks without energy cooperation were considered in the literature for various models. In [3], the optimal power allocation minimizing completion time of a file was found for an energy harvesting node with infinite battery. Optimal transmission policies for short-term throughput maximization and for completion time minimization were found for finite battery nodes in [4]. A directional water-filling algorithm was shown to yield the optimal policy in [5], [6] for the fading channel. Multiterminal models such as broadcast, multiple access, two-hop, and interference channels [7], [8], [9], [10], [11] were studied next. An insight that arose from these studies was that piecewise constant powers found by variations of the directional water-filling algorithm yield the optimum policy.

For networks with intermittent energy availability, energy cooperation among nodes is a desirable aspect to improve performance. For energy harvesting wireless networks, this is only relevant when energy transfer is wireless. Mid-range wireless energy transfer with magnetic coupling was studied in [12], [13] with a promising efficiency reaching $40 \%$, while shorter range inductive coupling already being used in RFID systems [14]. Transferring energy and information jointly has also been studied in [15] for an inductively coupled model and in [16] for a binary energy exchange model. Energy cooperation capability in short and mid-range introduces a new dimension for power allocation in energy harvesting networks. Energy transfer among energy harvesting wireless nodes was first studied in [1] for a two-hop network with a fullduplex infinite-buffer relay capable of uni-directional energy transfer from the source node to the relay node. Reference [2] considered the Gaussian two-way and two-user multiple access channels with uni-directional energy cooperation, proposing a two dimensional directional water-filling algorithm with meters to obtain the weighted sum-rate maximizing policy.

In this paper, we revisit the energy harvesting and energy cooperating Gaussian multiple access and two-way channels in [2] and extend the model to include bi-directional energy transfer. We first show that utilizing an equivalent set of energy transfer efficiency values allows us to restrict each node to either only send or only receive energy at any time without loss of optimality. We find that to obtain the jointly optimal transmission and energy transfer policy that maximizes the sum-rate, it is sufficient to find the energy transfer policy for each time slot and the consumed power allocation policy across the time slots. This is established by restricting the feasible policy set to procrastinating power policies, which we show to include at least one optimal power policy. For the multiple access channel, we observe that the optimal energy transfer policy for each node is to either transfer all of its energy, or transfer none at all; and that this is determined only by the energy transfer efficiency values of the node. We 


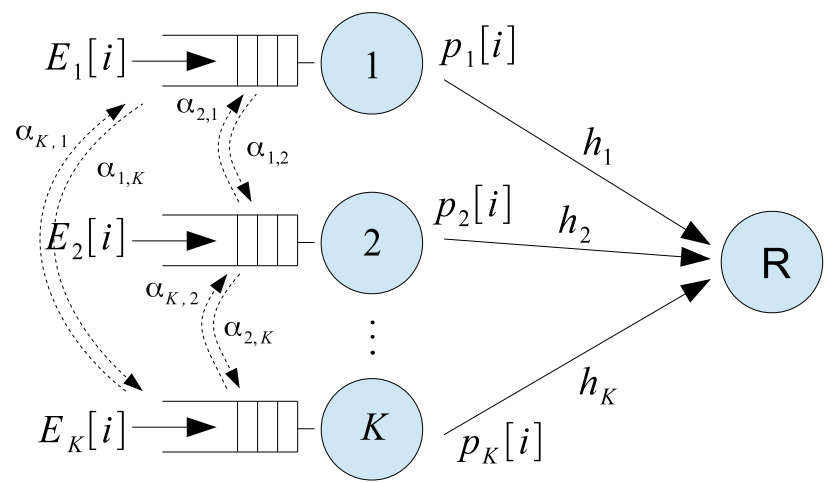

Fig. 1. K-transmitter multiple access channel with energy harvesting transmitters and energy cooperation.

thus show that the optimal power allocation problem for the energy cooperating MAC reduces to the single-link energy harvesting problem studied in [3] and the solutions can be found for a MAC with any number of transmitters. For the two-way channel, the power allocation problem is solved using a generalized iterative water-filling algorithm [17]. In this case, we observe that whenever a nonzero energy transfer is optimal, the optimal power allocation has a water-filling interpretation with two non-mixing fluids representing the powers of the two nodes. We demonstrate through 3-user MAC and TWC simulations that energy cooperation can provide significant improvements to the sum-capacity when some nodes in the network are energy deprived. We also demonstrate that in some cases, bi-directional energy cooperation can strictly outperform uni-directional energy cooperation of [1], [2].

\section{System Model and Problem Definition}

We consider the energy harvesting and energy cooperating multiple access and two-way channels shown in Figures 1 and 2 respectively. The multiple access channel consists of $K \geq 2$ energy harvesting transmitters conveying independent messages to the receiver $R$. In the two-way channel, energy harvesting transmitters 1 and 2 transmit independent messages to each other over a shared medium. In both models, all transmitters are capable of bi-directional energy transfer, allowing them to cooperate with any other transmitter by transferring a portion of their energy.

\section{A. Energy Cooperation Model}

A time slotted energy model with slot length $T$ is adopted without loss of generality. Throughout the paper, the subscripts $k, j$ and $\ell$ denote node indices, and $[i]$ and $[n]$ denote time slots. Transmitter $k$ harvests energy in packets of size $E_{k}[i]$ at the beginning of time slot $i$, and stores it in its energy storage device, referred to as its battery. We consider the case where the battery of each node is sufficiently large to avoid any overflow as in [1], [2], [3]. The energy harvests are known non-causally before transmission, and thus an optimal offline power allocation policy is to be found. Parallel to the notation in [1], [2], at the beginning of each time slot, transmitter $k$

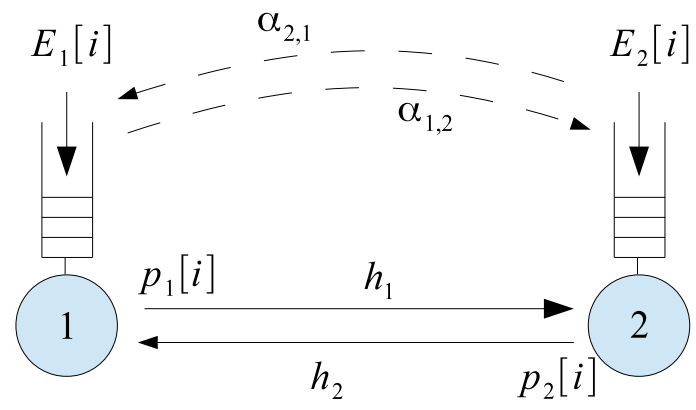

Fig. 2. Two-way channel with energy harvesting transmitters and energy cooperation.

chooses to transfer $\delta_{k, j}[i]$ amount of energy to transmitter $j$, where the transfer has an end-to-end efficiency of $\alpha_{k, j} \geq 0$. Hence, having energy $E_{k}^{-}[i]$ and $E_{j}^{-}[i]$ in their battery before the energy transfer, when transmitter $k$ transfers an energy of $\delta_{k, j}[i]$ to transmitter $j$, the energy available to both users immediately after transfer can be expressed as

$$
E_{k}[i]=E_{k}^{-}[i]-\delta_{k, j}[i], \quad E_{j}[i]=E_{j}^{-}[i]+\alpha_{k, j} \delta_{k, j}[i] .
$$

In time slot $i$, the energy available to node $k$ is governed by (i) the harvested energy, $E_{k}[i]$; (ii) the energy transferred to other nodes, $\delta_{k, j}[i]$ for all $j \neq k$; (iii) the energy received from other nodes, $\alpha_{j, k} \delta_{j, k}[i]$ for all $j \neq k$; and (iv) the energy consumed for transmission. Denoting the average power allocated to transmission at node $k$ in the $i^{\text {th }}$ time slot as $p_{k}[i]$, the energy stored in the battery of node $k$ at the end of the $i^{\text {th }}$ time slot is expressed as

$$
E_{k}^{b a t}[i]=\sum_{n=1}^{i}\left(E_{k}[n]+\sum_{j=1}^{K}\left(\alpha_{j, k} \delta_{j, k}[n]-\delta_{k, j}[n]\right)-p_{k}[n] T\right)
$$

where the self-transfer term $\delta_{k, k}[i]=0$ and its efficiency $\alpha_{k, k}=1$ by definition. For a transmit duration of $N$ time slots, the physical constraints on this model include the nonnegativity of transmit powers and transferred energy, i.e.,

$$
p_{k}[i] \geq 0, \quad \delta_{k, j}[i] \geq 0, \quad j, k=1, \ldots, K, \quad i=1, \ldots, N,
$$

and having sufficient energy to carry out both the transfer and the transmission within each time slot, referred to as energy causality [3], [4], [5]. Since a non-negative energy remaining in a node's battery implies energy causality, this condition is expressed as

$$
\sum_{n=1}^{i}\left(E_{k}[n]+\sum_{j=1}^{K}\left(\alpha_{j, k} \delta_{j, k}[n]-\delta_{k, j}[n]\right)-p_{k}[n] T\right) \geq 0
$$

for node $k$ in time slot $i$. The constraints in (3) and (4) describe the feasible set of power policies, i.e., transmit power allocation vectors, $\mathbf{p}_{k}=\left(p_{k}[1], \ldots, p_{k}[n]\right)$, and energy transfer vectors from $k$ to $j$ in all time slots, $\mathbf{d}_{k, j}=\left(\delta_{k, j}[1], \ldots, \delta_{k, j}[n]\right)$. 


\section{B. Energy Harvesting MAC with Energy Cooperation}

We first consider the Gaussian multiple access channel (MAC) [18] with $K$ energy harvesting and energy cooperating transmitters, shown in Figure 1. In this model, the channel output $Y$ is given by

$$
Y=\sqrt{h_{1}} X_{1}+\sqrt{h_{2}} X_{2}+\ldots+\sqrt{h_{k}} X_{K}+N
$$

where $N$ is the Gaussian noise with power $\sigma_{N}^{2}, X_{k}$ is the channel input of transmitter $k$, and $h_{k}$ 's are the channel coefficients, $k=1, \ldots, K$. Without loss of generality, we consider a normalized model with $h_{k}=1$ and $\sigma_{N}^{2}=1$, which can be established by scaling channel output $Y$ with $\sigma_{N}$ and transmit power of node $k$ by $h_{k} / \sigma_{N}^{2}$ for all $k=1, \ldots, K$.

Remark 1: Note that the scaling of transmit powers requires scaling the harvested energy packets $E_{k}[i]$ by $h_{k} / \sigma_{N}^{2}$ and energy transfer efficiency values $\alpha_{k, j}$ by $h_{j} / h_{k}$. This may result in $\alpha_{k, j}>1$ or $\alpha_{j, k}>1$ for some $(j, k)$ pair even though the physical energy transfer efficiency is less than 1 . Therefore, the only restriction on $\alpha_{k, j}$ in our model is nonnegativity, i.e., $\alpha_{k, j}>1$ does not imply energy transfer with efficiency greater than 1 in the physical sense, but is an artifact of normalization.

The sum-capacity of the Gaussian MAC is given by

$$
C_{S}^{M A C}=\frac{1}{2} \log \left(1+\sum_{k=1}^{K} p_{k}\right),
$$

where $p_{k}$ is the average transmit power constraint of node $k$.

\section{Energy Harvesting TWC with Energy Cooperation}

Next, consider the Gaussian two-way channel [19], [20] with energy harvesting and cooperating nodes, shown in Figure 2. In this model, the channel outputs observed by nodes 1 and 2 are given by

$$
\begin{aligned}
& Y_{1}=X_{1}+\sqrt{h_{2}} X_{2}+N_{1}, \\
& Y_{2}=X_{2}+\sqrt{h_{1}} X_{1}+N_{2},
\end{aligned}
$$

where $N_{k}$ is the Gaussian noise with power $\sigma_{k}^{2}$ at node $k$. Since node $k$ knows its own channel input $X_{k}$, the respective term can be canceled, and the model reduces to two parallel AWGN channels. Similar to the MAC case in Section II-B, receiver noise powers and channel coefficients can be normalized. Consequently, Remark 1 also applies to this model, i.e., normalization may yield $\alpha_{k, j}>1$ even though the physical transfer efficiency is less than unity. After the normalization, we can express the sum-capacity of this channel as

$$
C_{S}^{T W C}\left(p_{1}, p_{2}\right)=\frac{1}{2} \log \left(1+p_{1}\right)+\frac{1}{2} \log \left(1+p_{2}\right)
$$

where $p_{1}$ and $p_{2}$ are the average power constraints of the transmitters.

\section{Problem Formulation}

The sum-capacities of the two channels, specified in Sections II-B and II-C, give us the maximum sum-rate achievable within a single time slot for the set of transmit powers $p_{k}[i]$, $k=1, \ldots, K$. The sum-capacity of the energy harvesting network with energy transfer is defined as the maximum average sum-rate attained over the duration of transmission, where the transmit powers and energy transfers in each time slot are to be optimized. For a deadline of $N$ slots, with the constraints in (3) and (4), this sum-capacity problem for both models can be expressed as

$$
\begin{aligned}
\max _{\mathbf{p}_{k}, \mathbf{d}_{k, j}} & \frac{1}{N} \sum_{n=1}^{N} C_{S}\left(p_{1}[n], \ldots, p_{K}[n]\right), \\
\text { s.t. } & \delta_{k, j}[i] \geq 0, p_{k}[i] \geq 0, E_{k}^{\text {bat }}[i] \geq 0, \\
k=1, \ldots, K, \quad j & =1, \ldots, K, \quad i=1, \ldots, N
\end{aligned}
$$

where $p_{k}[n]$ is the transmit power of node $k$ in time slot $n$, $C_{S}=C_{S}^{M A C}$ for the MAC and $C_{S}=C_{S}^{T W C}$ for the TWC. We next prove that the problem in (10) can be decomposed and the energy transfer problem can be solved on a slot-by-slot basis.

\section{Properties of Optimal Policies}

In this section, we consider a class of policies conforming to additional constraints on energy transfer and transmission power, and show that this class contains at least one globally optimal policy. These properties will allow us to rewrite the problem in terms of consumed powers in Section III-C, and ultimately simplify the solution.

\section{A. Routing Energy Transfers}

We first point out that in a model with two or more transmitters, it is possible to have multiple energy transfer paths between any two nodes. For example, node 1 can transfer energy to node 2 over the transfer link with efficiency $\alpha_{1,2}$, or through node 3 with efficiency $\alpha_{1,3} \alpha_{3,2}$. Hence, finding the best energy transfer path is embedded in our problem. The optimal energy routing involves choosing the transfer path with the highest effective efficiency, since using any other path results in an energy loss that can be avoided. This path can be calculated from $\left\{\alpha_{k, j}\right\}$ only, independent from harvested and transferred energy amounts. To account for the optimal energy transfer routing while reducing the size of the feasible set in (10), we define an equivalent set of energy transfer efficiency values, $\bar{\alpha}_{k, j}$, such that

$$
\bar{\alpha}_{k, j}=\max \alpha_{k, e_{1}} \alpha_{e_{1}, e_{2}} \ldots \alpha_{e_{m}, j}
$$

where $\left(e_{1}, \ldots, e_{m}\right)$ is any ordered subset of node indices excluding $k$ and $j$. Thus, $\left(k, e_{1}, \ldots, e_{m}, j\right)$ is any feasible energy transfer path without a cycle, including a direct transfer $(k, j)$. The maximization in (11) is taken over all possible such paths. This definition allows us to treat any energy transferred from node $k$ to node $j$ as if it is transferred over a direct transfer link with the best possible efficiency. In the optimal policy found using the effective $\bar{\alpha}_{k, j}$ 's, if any energy 
is transferred between two nodes with $\bar{\alpha}_{k, j} \neq \alpha_{k, j}$, then the physical energy transfers are found to be transferred through the path yielding $\bar{\alpha}_{k, j}$. When this equivalent set of efficiency values is used, the following property for the optimal power allocation is observed:

Lemma 1: There exists an optimal policy where any node $k$ is either sending energy only, or receiving energy only at a particular time, i.e., either $\delta_{k, j}[i]=0$ or $\delta_{j, k}[i]=0$ for all $j \in\{1, \ldots, K\}$ in any time slot $i$.

Proof: Let some node $k$ receive energy from node $j$ and transfer energy to node $\ell$ in time slot $i$, i.e., $\delta_{j, k}[i]>0$ and $\delta_{k, \ell}[i]>0$. A policy with the same transmit power vector $\mathbf{p}_{\mathbf{k}}$ can be found by increasing $\delta_{j, \ell}[i]$ by $\min \left\{\delta_{j, k}[i], \delta_{k, \ell}[i] / \bar{\alpha}_{j, k}\right\}$, and decreasing $\delta_{j, k}[i]$ and $\delta_{k, \ell}[i]$ by $\delta_{j, \ell}[i]$ and $\bar{\alpha}_{j, k} \delta_{j, \ell}[i]$ respectively. This ensures that either $\delta_{j, k}[i]=0$ or $\delta_{k, \ell}[i]=0$. Repeating for all violations of the conditions yields a policy satisfying the conditions of the Lemma.

Lemma 1 provides the insight that when $\bar{\alpha}_{k, j}$ are used, each node can be restricted to either transfer or receive energy at any time instant. This allows us to decrease the size of the search space for energy transfers, and provide useful properties for the feasible power allocations. This also rules out policies with simultaneous bi-directional transfer between two nodes, or policies with cycles on energy transfer paths, which are trivially suboptimal. With a slight abuse of notation, we denote the equivalent set of efficiency values as $\alpha_{j, k}$ in the sequel, remembering that the physical energy transfers can be found as discussed above.

\section{B. Procrastinating Policies}

Definition 1: A power policy consisting of transmit powers $\left(\mathbf{p}_{k}\right)_{k=1, \ldots, K}$ and energy transfers $\left(\mathbf{d}_{k, j}\right)_{k, j=1, \ldots, K}$ is a procrastinating policy if

$$
p_{j}[i] T \geq \sum_{k=1}^{K} \alpha_{k, j} \delta_{k, j}[i]
$$

is satisfied for all $j=1, \ldots, K$ and $i=1, \ldots, N$.

The definition implies that in any time slot, the energy consumed for transmission in node $j$ is greater than the total energy transferred to that node in that time slot. The procrastination here is observed at the energy transferring nodes, which delay their energy transfer unless the receiving node intends to use it immediately. Next, we show that there exists at least one globally optimum procrastinating power policy.

Lemma 2: There exists at least one feasible procrastinating policy that achieves the sum-capacity in (10).

Proof: Assume that an optimum policy, $\left(\mathbf{p}_{k}^{*}\right)_{k=1, \ldots, K}$, $\left(\mathbf{d}_{k, j}^{*}\right)_{k, j=1, \ldots, K}$, is not a procrastinating policy, i.e., let $p_{j}^{*}[i] T<\sum_{k=1}^{K} \alpha_{k, j} \delta_{k, j}^{*}[i]$ for some $i$ and $j$. Then, one can scale all $\delta_{k, j}^{*}[i]$ for $k=1, \ldots, K$ with $0 \leq \sigma<1$, where $\sigma=p_{j}^{*}[i] T /\left(\sum_{k=1}^{K} \alpha_{k, j} \delta_{k, j}^{*}[i]\right)$, and obtain the energy transfers $\delta_{k, j}^{\prime}[i]=\sigma \delta_{k, j}^{*}[i]$, which satisfy the procrastinating condition (12) in time slot $i$. Next, the energy transfers in the following time slot can be redefined as $\delta_{k, j}^{\prime}[i+1]=$ $\delta_{k, j}^{*}[i+1]+(1-\sigma) \delta_{k, j}^{*}[i]$. This ensures that all energy causality conditions after time slot $i$ are satisfied. Hence, the policy $\left(\mathbf{p}_{k}^{*}, \mathbf{d}_{k, j}^{\prime}\right)$ is feasible. Notice that this procedure represents delaying energy transfers if they are not immediately needed. Since this procedure does not affect the energy transfers before time slot $i$ or energy transfers received by other nodes, it can be employed for each time slot $i=1, \ldots, N$, and repeated for each node separately, yielding a policy that satisfies (12) for all $i$ and $k$. Hence, the sum-capacity can also be achieved with an equivalent procrastinating policy.

Lemma 2 shows that by delaying energy transfers in each time slot, i.e., procrastinating when possible, a feasible and optimum procrastinating policy can always be found.

\section{Decomposition of the Problem}

Restricting our feasible set to procrastinating policies that satisfy the conditions in Lemma 1, we define consumed powers $\overline{\mathbf{p}}_{\mathbf{k}}=\left[\bar{p}_{k}[1], \bar{p}_{k}[2], \ldots, \bar{p}_{k}[n]\right] \geq 0$ as

$$
\bar{p}_{k}[i]=p_{k}[i]+\frac{1}{T} \sum_{j=1}^{K} \delta_{k, j}[i]-\alpha_{j, k} \delta_{j, k}[i] .
$$

Due to Lemma 1 and non-negativity of $p_{k}[i]$, a procrastinating policy that satisfies (12) also satisfies

$$
T \bar{p}_{k}[i] \geq \sum_{j=1}^{K} \delta_{k, j}[i],
$$

which is sufficient to ensure non-negativity of $p_{k}[i]$ given the non-negativity of $\overline{\mathbf{p}}_{k}$ and $\mathbf{d}_{k, j}$. Hence, the problem in (10) can be rewritten, ignoring the constant $\frac{1}{N}$, as

$$
\begin{aligned}
\max _{\overline{\mathbf{p}}_{k}, \mathbf{d}_{k, j}} & \sum_{i=1}^{N} C_{s}\left(\bar{p}_{k}[i]-\frac{1}{T} \sum_{j=1}^{K}\left(\delta_{k, j}[i]-\alpha_{j, k} \delta_{j, k}[i]\right)\right), \\
\text { s.t. } & \sum_{n=1}^{i} E_{k}[n]-\bar{p}_{k}[n] T \geq 0, \quad \overline{\mathbf{p}}_{k} \geq 0 \\
& T \bar{p}_{k}[i] \geq \sum_{j=1}^{K} \delta_{k, j}[i], \quad \mathbf{d}_{k, j} \geq 0 \\
& k=1, \ldots, K, \quad j=1, \ldots, K, \quad i=1, \ldots, N .
\end{aligned}
$$

where the first term in (15b) is the energy causality constraint and $(15 \mathrm{c})$ is the non-negativity of $p_{k}[i]$ and $\delta_{k, j}[i]$. The energy causality constraints are now independent of $\mathbf{d}_{k, j}$ and the constraints on $\delta_{k, j}[i]$ are separated among time slots, $i$. Thus, the problem can be decomposed as

$$
\begin{aligned}
\max _{\overline{\mathbf{p}}_{k}} & \sum_{i=1}^{N} \max _{\mathbf{d}_{k, j},(15 c)} C_{S}\left(\bar{p}_{k}[i]-\frac{1}{T} \sum_{j=1}^{K}\left(\delta_{k, j}[i]-\alpha_{j, k} \delta_{j, k}[i]\right)\right) \\
\text { s.t. } & \sum_{n=1}^{i} E_{k}[n]-\bar{p}_{k}[n] T \geq 0, \quad \overline{\mathbf{p}}_{k} \geq 0 \\
& k=1, \ldots, K, \quad i=1, \ldots, N .
\end{aligned}
$$


Note that the inner maximization is only over $\delta_{k, j}[i]$, and is subject to the respective constraints in (15c). This implies that the optimal energy transfer can be calculated independently for each time slot given a set of consumed powers $\left\{\bar{p}_{k}[i]\right\}_{k=1, \ldots, K}$. Let the sum-capacity achieved in a timeslot with fixed consumed powers $\left\{\bar{p}_{k}[i]\right\}_{k=1, \ldots, K}$ allowing energy transfer be $C^{*}\left(\bar{p}_{k}[i]\right)$. Substituting $C^{*}$ in (16) yields a problem that has the same structure as the scheduling problems previously considered for energy harvesting networks without energy transfer [3], [4], [5], and a generalized iterative waterfilling algorithm can be used to find the optimal policy as long as $C^{*}$ is jointly concave in consumed powers [17]. The joint concavity of $C^{*}$ can readily be shown by a time-sharing argument, where any point violating concavity can be strictly improved by time-sharing [11].

\section{Optimal Policy For MAC With ENERgy COOPERATION}

Substituting the sum-capacity $C_{S}^{M A C}$ (6) in (16), the inner maximization for time slot $i$ can be simplified as

$$
\begin{aligned}
\max & \sum_{k=1}^{K} \sum_{j=1}^{K} \delta_{k, j}[i]\left(\alpha_{k, j}-1\right) \\
\text { s.t. } & 0 \leq \delta_{k, j}[i] \leq \bar{p}_{k}[i] T, \quad k, j=1,2, \ldots, K k \neq j .
\end{aligned}
$$

which is obtained by omitting constant terms and the logarithm for being monotonic. The solution to (17) is found as a decision based only on $\alpha_{k, j}$ for each $k$ and $j$. If $\max _{j} \alpha_{k, j}=$ $\alpha_{k, j^{*}}>1$ for node $k$, then node $k$ transfers all of its allocated energy to node $j^{*}$ in each time slot, i.e., $\delta_{k, j^{*}}[i]=\bar{p}_{k}[i] T$ for all $i$. Otherwise, $k$ never transfers energy to any user, i.e., $\delta_{k, j}[i]=0$ for all $j$ and $i$.

Notice that this result also conforms to Lemma 1. Let $\max \alpha_{k, j}=\alpha_{k, j^{*}}>1$, and node $k$ transfers all of its energy to node $j^{*}$. In this case, we can show node $k$ does not receive energy from any other node, and node $j^{*}$ does not transfer energy to any other node. For node $\ell, \ell \neq k, j^{*}$ to transfer its energy to node $k$, we need $\max _{j} \alpha_{\ell, j}=\alpha_{\ell, k}$. However, this cannot hold since

$$
\alpha_{\ell, k}<\alpha_{\ell, k} \alpha_{k, j^{*}} \leq \alpha_{\ell, j^{*}} \leq \max _{j} \alpha_{\ell, j}
$$

where the second inequality follows from (11). Similarly, for node $j^{*}$ to transfer energy to node $\ell$, we need $\alpha_{j^{*}, \ell}>1$. This is not possible since $\alpha_{k, \ell} \geq \alpha_{k, j^{*}} \alpha_{j^{*}, \ell}>\alpha_{k, j^{*}}$, which contradicts $\max _{j} \alpha_{k, j}=\alpha_{k, j^{*}}$.

Since $\alpha_{k, j}$ and $\alpha_{j, k}$ are constant and cannot be greater than 1 simultaneously, the direction of energy transfer is fixed throughout the transmission. As a result, in a static multiple access channel with energy harvesting and energy transfer, a carefully chosen uni-directional energy transfer capability is sufficient to achieve bi-directional energy transfer capacity. However if channel coefficients or energy transfer efficiency values are time varying, energy transfer in both directions would need to be utilized.

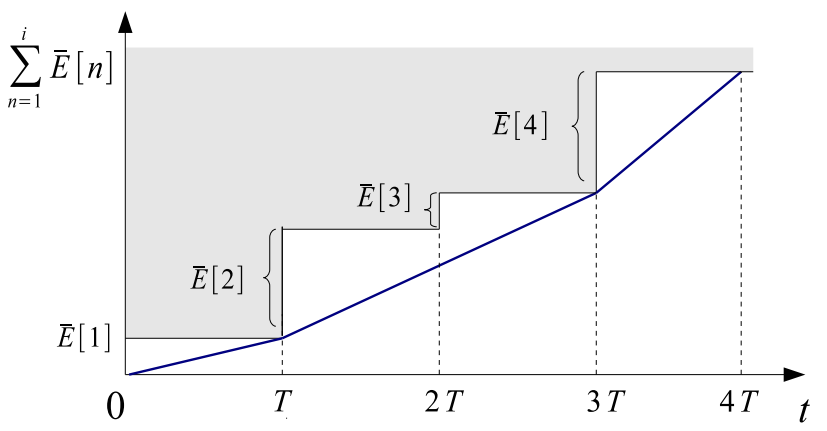

Fig. 3. Optimal sum-power policy for an energy cooperating MAC for a deadline of 4 time slots.

The power allocation problem among time slots is consequently simplified as follows: if one transmitter ends up receiving all the energy from the other nodes, the problem trivially reduces to a single link studied in [3], [4]. If two or more transmitters are transmitting, the problem reduces to the sum-rate problem solved in [8] or its multi-user extension. In this case, the solution is found as if all transmitting nodes had a single pool of energy, which also reduces to the single link problems in [3], [4] with joined energy harvests. Let

$$
a_{k}=\max _{j} \alpha_{k, j} \text {. }
$$

Then, the optimal power allocation policy for the MAC with energy transfer can be found as the solution to the single user problem with arrivals

$$
\bar{E}[i]=\sum_{k=1}^{K} a_{k} E_{k}[i],
$$

which is solved by constructing the cumulative energy curve and finding the shortest path from the initial point, i.e., origin, to the staircase at the $N^{t h}$ time slot [3]. An example is shown in Figure 3 for $N=4$, where the staircase is the sum of $\bar{E}[i]$ for all $k$ and from 0 to current time slot $i$. A feasible energy policy, that is the cumulative energy spent up to some time slot, cannot trespass to the shaded region due to energy causality conditions. The optimal energy policy in this example is shown in blue, from which the sum power can be found. Once the optimal sum power policy is found, the transmitting users are free to choose any set of transmit powers that yield this sum power.

\section{OPtimal POLICY FOR TWC WITH ENERGY COOPERATION}

Substituting (9) in (16), the inner maximization over energy transfers in time slot $i$ is simplified as

$$
\begin{aligned}
& \max \left(1+\bar{p}_{1}[i]-\delta_{1,2}[i]+\alpha_{2,1} \delta_{2,1}[i]\right) . \\
&\left(1+\bar{p}_{2}[i]-\delta_{2,1}[i]+\alpha_{1,2} \delta_{1,2}[i]\right) \\
& \text { s.t. } 0 \leq \delta_{k, j}[i] \leq \bar{p}_{k}[i] T, \quad k, j=1,2, k \neq j .
\end{aligned}
$$

Notice that from Lemma 1, we know that the energy flow may only be in one direction at a given time. Hence, we partition the feasible space into two subspaces with $\delta_{1,2}[i]=0$ and 
$\delta_{2,1}[i]=0$, solve the problem separately for each subspace, and find the solution as the maximum of the two results. The two subspaces admit the candidates for the optimal energy transfer vector,

$$
\hat{\delta}_{k, j}[i]=\max \left(\frac{1}{2}\left(1+\bar{p}_{k}[i]-\frac{1+\bar{p}_{j}[i]}{\alpha_{k, j}}\right), 0\right),
$$

where $k, j=1,2$ and $j \neq k$. If both candidates are zero, as is the case for close values of $\bar{p}_{k}[i]$ or the case for very small transfer efficiency in both directions, the optimal policy does not involve any energy transfer. If only one candidate is positive, then the solution is given by $\left(\hat{\delta}_{1,2}[i], \hat{\delta}_{2,1}[i]\right)$. Finally, if both are positive, then the direction maximizing $\alpha_{k, j}\left(\hat{\delta}_{k, j}[i]\right)^{2}$ is chosen as the transfer direction and the other one is set to zero.

Denoting the energy transferring node as $\ell \in\{0,1,2\}$ and the energy receiving node as $\bar{\ell} \in\{1,2\}, \bar{\ell} \neq \ell$, with $\ell=0$ corresponding to no energy transfer, the sum-capacity achieved with energy transfer in time slot $i$ is found as

$C^{*}\left(\bar{p}_{k}[i]\right)= \begin{cases}\log \left(\frac{\sqrt{\alpha_{\ell, \bar{\ell}}}}{2}\left(1+\bar{p}_{\ell}[i]+\frac{1+\bar{p}_{\bar{\ell}}[i]}{\alpha_{\ell, \bar{\ell}}}\right)\right) & \ell \in\{1,2\} \\ C_{S}^{T W C}\left(p_{1}[i], p_{2}[i]\right) & \ell=0\end{cases}$

The power allocation problem can subsequently be solved by substituting above expression in (16), and employing the generalized iterative water-filling algorithm in [17]. This yields the water-levels

$$
v_{1}[i]= \begin{cases}2\left(1+\bar{p}_{1}[i]\right) & \ell=0 \\ 1+\bar{p}_{1}[i]+\frac{1+\bar{p}_{2}[i]}{\alpha_{1,2}} & \ell=1 \\ \alpha_{2,1}\left(1+\bar{p}_{2}[i]+\frac{1+\bar{p}_{1}[i]}{\alpha_{2,1}}\right) & \ell=2\end{cases}
$$

for the iterations of node 1 , and

$$
v_{2}[i]= \begin{cases}2\left(1+\bar{p}_{2}[i]\right) & \ell=0 \\ \alpha_{1,2}\left(1+\bar{p}_{1}[i]+\frac{1+\bar{p}_{2}[i]}{\alpha_{1,2}}\right) & \ell=1 \\ 1+\bar{p}_{2}[i]+\frac{1+\bar{p}_{1}[i]}{\alpha_{2,1}} & \ell=2\end{cases}
$$

for the iterations of node 2. An example for $N=4$ and $T=1$ with $\alpha_{1,2}=\alpha_{2,1}=0.5$ is given in Figure 4 , where the optimal water levels are shown in blue for node 1 and green for node 2 for energy arrivals $E_{1}[1]=2, E_{1}[2]=5$, $E_{2}[2]=4$ and $E_{2}[4]=7$. In the first time slot, $\bar{p}_{1}[1]=2$ and $\bar{p}_{2}[1]=0$ gives the candidates $\left(\hat{\delta}_{1,2}[1], \hat{\delta}_{2,1}[1]\right)=(1,0)$. Therefore, $\ell=1$ in time slot 1 , i.e., node 1 sends 1 unit of energy to node 2 , yielding the water levels shown. In time slots $i=2,3$, the candidates are $\left(\hat{\delta}_{1,2}[i], \hat{\delta}_{2,1}[i]\right)=(0,0)$, and thus no node transfers any energy. Finally in time slot 4 , candidates become $\left(\hat{\delta}_{1,2}[4], \hat{\delta}_{2,1}[4]\right)=(0,2)$, and node 2 transfers 2 units of energy to node 1 . The energy transfers in each slot are denoted with red arrows. The resulting water levels conform to the directional water-filling properties such as constant water levels among slots with level increases only when the respective node is out of energy.

For the two-way channel, the insight arises that in time slots with nonzero energy transfer, i.e., $\ell=1,2$, the optimal energy

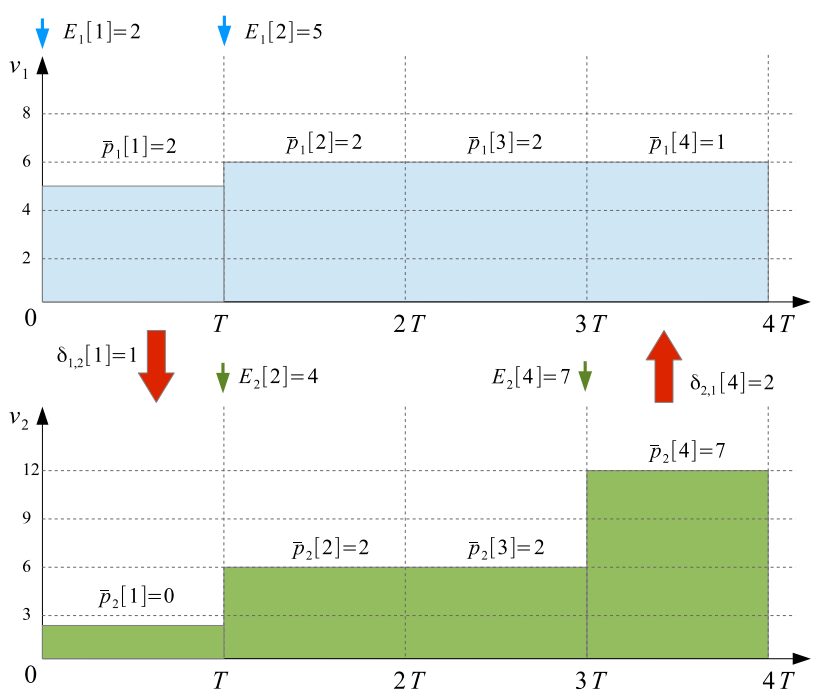

Fig. 4. Optimal water levels found by iterative generalized water-filling with water-levels defined in (23) and (24).

transfer yields $\alpha_{\ell, \bar{\ell}}\left(1+p_{\ell}[i]\right)=\left(1+p_{\bar{\ell}}[i]\right)$, and the resulting $C^{*}\left(\bar{p}_{k}[i]\right)$ is a logarithmic function concave in $\bar{p}_{\ell}[i]+b \bar{p}_{\bar{\ell}}[i]$ for some $b$. Consequently, the generalized water-levels used in the iterative directional water-filling algorithm are linearly related, reducing the algorithm to a non-iterative directional water-filling algorithm with water levels given by

$$
v[i]=\frac{\sqrt{\alpha_{\ell, \bar{\ell}}}}{2}\left(1+\bar{p}_{\ell}[i]+\frac{1+\bar{p}_{\bar{\ell}}[i]}{\alpha_{\ell, \bar{\ell}}}\right), \quad \ell, \bar{\ell} \in\{1,2\}, \ell \neq \bar{\ell} .
$$

We remark that omitting the constant terms in (25), directional water-filling allows forward water-flow to equalize $\bar{p}_{\ell}[i]+\frac{\bar{p}_{\bar{C}}[i]}{\alpha_{\ell, \bar{\ell}}}$ among time slots. This is water-filling of two non-mixing fluids with the power of node $\bar{\ell}$ scaled by $\alpha_{\ell, \bar{\ell}}$.

\section{NumeriCAL RESUlTS}

To demonstrate the sum-capacity improvement in MAC with energy cooperation, we present simulation results for a three transmitter MAC. For channel coefficients $h_{1}=-100 d B$, $h_{2}=-105 d B$ and $h_{3}=-110 d B$, receiver noise density $N_{0}=10^{-19} \mathrm{~W} / \mathrm{Hz}$, and a bandwidth of $1 \mathrm{MHz}$, we generate energy arrivals, uniformly distributed in $[0,1] \mathrm{mJ}$ for node 1 , and $\left[0, E_{h}\right] m J$ for nodes 2 and 3 in $N=100$ time slots of length $T=1 \mathrm{sec}$. The energy transfer coefficients are chosen as

$$
\left[\alpha_{k, j}\right]=\left[\begin{array}{ccc}
1 & 0.3 & 0.2 \\
0.1 & 1 & 0.4 \\
0.4 & 0 & 1
\end{array}\right]
$$

in accordance with the $40 \%$ efficiency achieved in [12], [13]. Note that this is a system where node 1 has a relatively better channel but is energy deprived. The sum-capacity as a function of peak harvest rates $E_{h}$ for nodes 2 and 3 is given in Figure 5 in comparison to the case without energy transfer and to the best effort scheme with constant transmit 


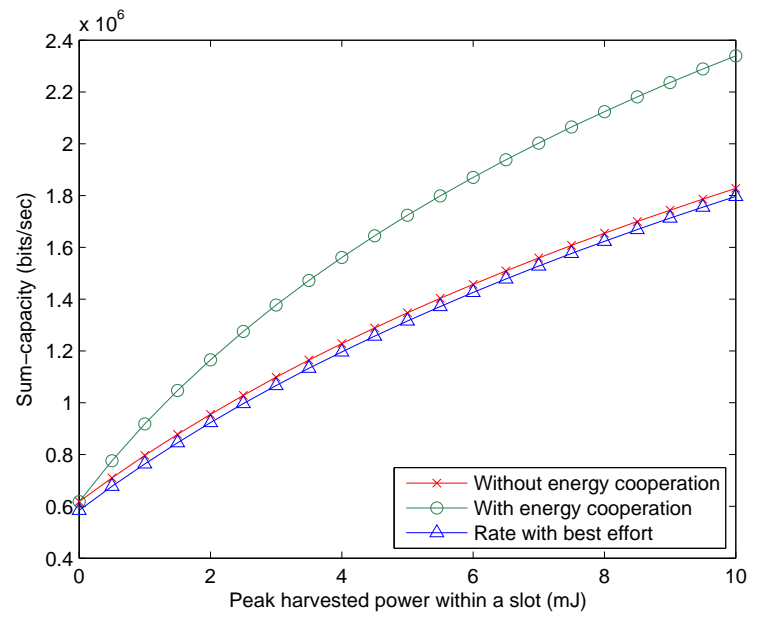

Fig. 5. Sum-capacity with varying $E_{h}$ for a 3 user MAC without and with energy transfer, and with constant power allocation.

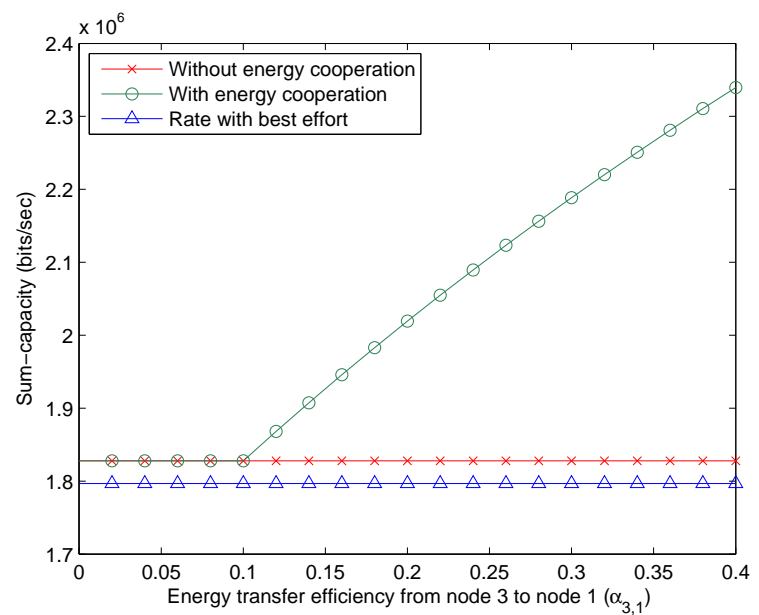

Fig. 6. Sum-capacity with $\alpha_{3,1}$ for a 3 user MAC. At $\alpha_{3,1}=0.1$, node 3 starts transferring its energy to node 1 .

power, which is known to approach capacity as $N \rightarrow \infty$ for infinite batteries [21]. A notable improvement is observed in this case, for which the optimal energy transfer policy involves node 3 to send all harvested energy to node 1 . To demonstrate the effect of energy transfer efficiency, sumcapacity values for the three policies as a function of $\alpha_{3,1}$ is plotted in Figure 6 for $E_{h}=10 \mathrm{~mJ}$. It is observed that node 3 transfers energy to node 1 for $\alpha_{3,1}>0.1$, achieving the improvement in Figure 5. Notice that since the optimal policy dictates energy transfer in only one direction, the unidirectional energy transfer considered in [2] either achieves the sum-capacity with bi-directional energy transfer, or the sumcapacity without energy transfer, depending on the direction of the optimal energy transfer found for the bi-directional model.

Next, we simulate the two-way channel with $\alpha_{1,2}=0.3$ and $\alpha_{2,1}=0.4$, with channel coefficients $h_{1}=-100 \mathrm{~dB}$ and $h_{2}=$ $-110 d B$. The remaining parameters $N_{0}, W, T$ and $N$ are unchanged. For $E_{2}[i]$ distributed uniformly in $[0,10] \mathrm{mJ}$, and

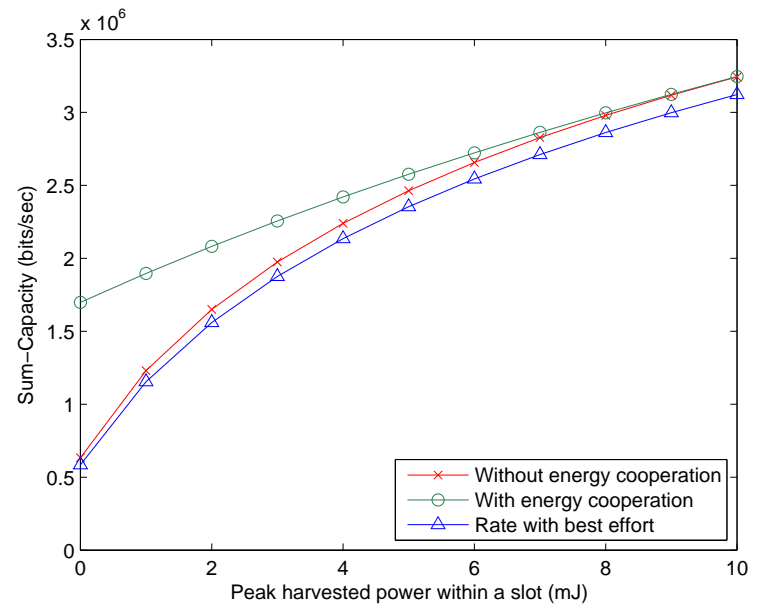

Fig. 7. Sum-capacity with varying $E_{h}$ for a TWC without and with energy transfer, and with constant power allocation.

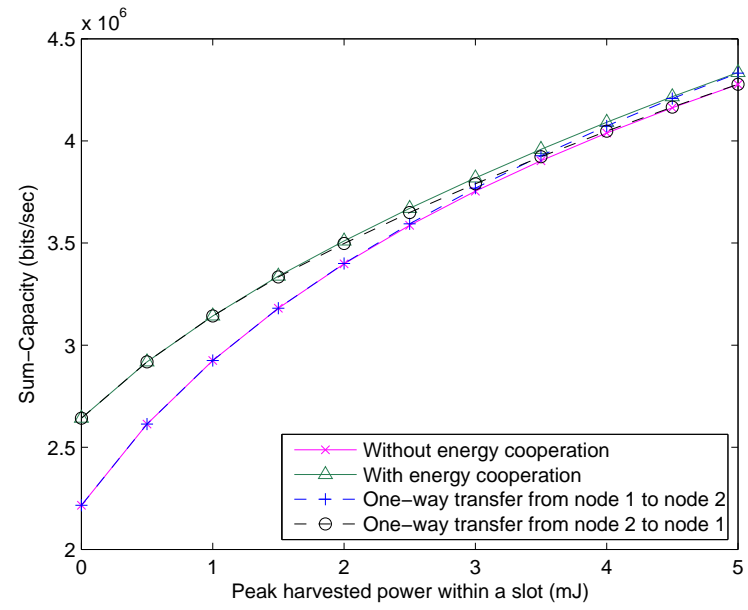

Fig. 8. Sum-capacity with varying $E_{h}$ for a TWC without and with unidirectional and bi-directional energy transfer.

$E_{1}[i]$ distributed uniformly in $\left[0, E_{h}\right] m J$, the sum-capacity results are plotted in Figure 7 against $E_{h}$. It is observed that the improvement with energy transfer is more significant when one node is energy deprived. Also notice that the improvement due to bi-directional energy transfer is increasing with $E_{h}$ as energy transferring node gets more energy as is the case in Figure 5, and is decreasing with $E_{h}$ as energy receiving node gets more energy as is the case in Figure 7.

Over many such experiments, it is observed that for the TWC, energy transfer is particularly beneficial when one node is energy deprived or has a bad channel. In such cases, the direction of energy transfer is fixed for the majority of the transmission. Thus, a uni-directional energy transfer with the correct direction is sufficient to recover sum-capacity. However, when nodes have similar parameters, it can be observed that uni-directional energy transfer may not be sufficient. An example is shown in Figure 8 for $h_{1}=h_{2}=-100 \mathrm{~d} B$, $E_{2}[i]$ distributed uniformly in $[0,10] m J, E_{1}[i]$ distributed 
uniformly in $\left[0, E_{h}\right] m J$, and $\alpha_{1,2}=\alpha_{2,1}=0.5$. It can be observed that for very low or sufficiently high $E_{h}$, a uni-directional energy transfer approaches bi-directional sumcapacity. However, in between, it is observed that energy transfer in both directions performs strictly better than either of the uni-directional models.

\section{CONCLUSION}

For the energy harvesting multiple access and two-way channels with bi-directional energy transfer, we have considered the problem of finding the sum-capacity by jointly optimizing transmit power and energy transfer policies. We found that for networks with more than two energy cooperating nodes, using an effective energy transfer efficiency derived from the most efficient path between the two nodes allow each node to be either in an energy transferring or energy receiving state. We have shown that the problem of power allocation among time slots and the problem of optimal energy transfer within each time slot can be solved separately. In the multiple access channel, we have shown the optimal energy transfer strategy to be independent of allocated powers or energy harvests, since a node chooses to either transfers all of its energy to another node or not transfer at all based on transfer efficiency values only. The power allocation problem is therefore reduced to the short-term throughput maximization problem for an energy harvesting single link without energy transfer, and its solution can be found by directional waterfilling. In the two-way case, the optimal energy transfer was found to be a function of two transfer candidates. Whenever a nonzero energy transfer is optimal, the power allocation was shown to have a water-filling interpretation with two nonmixing fluids. Simulations demonstrated a significant sumcapacity improvement with energy cooperation for a MAC with 3 users and for a TWC, particularly when one of the nodes is energy deprived. It is also observed that in some cases, bi-directional energy cooperation can outperform unidirectional energy transfer in either direction. Future work on energy harvesting networks with energy cooperation includes studying models with finite energy storage, data cooperation, and time-varying energy transfer efficiency values in offline and online settings.

\section{REFERENCES}

[1] B. Gurakan, O. Ozel, J. Yang, and S. Ulukus, "Energy cooperation in energy harvesting wireless communications," in Proceedings of IEEE International Symposium on Information Theory (ISIT),. IEEE, 2012, pp. $965-969$.

[2] energy cooperation," in 46th Asilomar Conference on Signals, Systems and Computers. Pacific Grove, CA: IEEE, Nov. 2012.

[3] J. Yang and S. Ulukus, "Optimal packet scheduling in an energy harvesting communication system," IEEE Transactions on Communications, vol. 60, no. 1, pp. 220-230, 2012.

[4] K. Tutuncuoglu and A. Yener, "Optimum transmission policies for battery limited energy harvesting nodes," IEEE Transactions on Wireless Communications, vol. 11, no. 3, pp. 1180-1189, Mar. 2012.

[5] O. Ozel, K. Tutuncuoglu, J. Yang, S. Ulukus, and A. Yener, "Transmission with energy harvesting nodes in fading wireless channels: Optimal policies," IEEE Journal on Selected Areas in Communications, vol. 29, no. 8, pp. $1732-1743$, Sep. 2011.
[6] C. Ho and R. Zhang, "Optimal energy allocation for wireless communications powered by energy harvesters," in Proceedings of IEEE International Symposium on Information Theory (ISIT), 2010, pp. 2368 2372.

[7] J. Yang, O. Ozel, and S. Ulukus, "Broadcasting with an energy harvesting rechargeable transmitter," IEEE Transactions on Wireless Communications, vol. 11, no. 2, pp. 571 -583, Feb. 2012.

[8] J. Yang and S. Ulukus, "Optimal packet scheduling in a multiple access channel with energy harvesting transmitters," Communications and Networks, Journal of, vol. 14, no. 2, pp. 140-150, 2012.

[9] D. Gunduz and B. Devillers, "Two-hop communication with energy harvesting," in Proceedings of the 4th International Workshop on Computational Advances in Multi-Sensor Adaptive Processing, (CAMSAP), Dec. 2011.

[10] O. Orhan and E. Erkip, "Energy harvesting two-hop networks: Optimal policies for the multi-energy arrival case," in 35th IEEE Sarnoff Symposium (SARNOFF). IEEE, 2012, pp. 1-6.

[11] K. Tutuncuoglu and A. Yener, "Sum-rate optimal power policies for energy harvesting transmitters in an interference channel," JCN Special Issue on Energy Harvesting in Wireless Networks, vol. 14, no. 2, pp. 151-161, April 2012.

[12] A. Kurs, A. Karalis, R. Moffatt, J. Joannopoulos, P. Fisher, and M. Soljačić, "Wireless power transfer via strongly coupled magnetic resonances," Science, vol. 317, no. 5834, pp. 83-86, 2007.

[13] A. Karalis, J. Joannopoulos, and M. Soljačić, "Efficient wireless nonradiative mid-range energy transfer," Annals of Physics, vol. 323, no. 1, pp. 34-48, 2008.

[14] R. Want, "An introduction to rfid technology," Pervasive Computing, IEEE, vol. 5, no. 1, pp. 25-33, 2006.

[15] P. Grover and A. Sahai, "Shannon meets tesla: wireless information and power transfer," in Information Theory Proceedings (ISIT), 2010 IEEE International Symposium on. IEEE, 2010, pp. 2363-2367.

[16] P. Popovski, A. Fouladgar, and O. Simeone, "Interactive joint transfer of energy and information," submitted to IEEE Transactions on Communications, 2012, available at arXiv:1209.6367.

[17] K. Tutuncuoglu and A. Yener, "Communicating with energy harvesting transmitters and receivers," in Proceedings of the 2012 Information Theory and Applications Workshop, ITA'12, Feb. 2012.

[18] R. Ahlswede, "Multi-way communication channels," in Proc. 2nd Int. Symp. Information Theory, 1971, pp. 23-52.

[19] C. Shannon, "Two-way communication channels," in Proc. 4th Berkeley Symp. Math. Stat. Prob, vol. 1, 1961, pp. 611-644.

[20] T. Han, "A general coding scheme for the two-way channel," Information Theory, IEEE Transactions on, vol. 30, no. 1, pp. 35-44, 1984.

[21] O. Ozel and S. Ulukus, "Information-theoretic analysis of an energy harvesting communication system," in IEEE 21st International Symposium on Personal, Indoor and Mobile Radio Communications Workshops (PIMRC Workshops), 2010, pp. 330-335. 